\title{
Cuadernos de economía
}

www. elsevier. es/ cesjef

ARTÍCULO

\section{Determinantes de la inversión en exploración de hidrocarburos: un análisis del caso argentino}

\section{Marina Yesica Recalde}

Departamento de Economía/ UNS, Instituto de Investigaciones Económicas y Sociales del Sur (IIESS)-CONICET-UNS

Recibido el 6 de diciembre de 2010; aceptado el 1 de febrero de 2011

\section{CÓDIGOS J EL}

Q43;

C13;

G11

\section{PALABRAS CLAVE}

Inversión en exploración; Coeficientes de correlación; Determinantes; Incertidumbre
Resumen En este trabajo se estudia el comportamiento cíclico de la inversión en exploración en el upstream de petróleo y gas natural en Argentina, y de algunas de las principales variables económicas de acuerdo con la metodología de Kydland y Prescott (1990). El fin del trabajo es realizar una aproximación a los determinantes de dicha inversión en un país en desarrollo. Algunos de los principales resultados cualitativos se relacionan con la falta de capacidad explicativa individual de las variables económicas, la relevancia de la incertidumbre y su impacto en la racionalidad de los agentes al momento de la toma de decisiones, en particular en países en desarrollo como el caso que aquí se analiza. Estos aspectos deben ser tomados particularmente en consideración al momento del análisis de la sustentabilidad del sistema energético y del diseño de la política energética, principalmente dado el rol del abastecimiento energético en el desarrollo socioeconómico.

(C) 2010 Asociación Cuadernos de Economía. Publicado por Elsevier España, S. L. Todos los derechos reservados.

\section{Determinants for investing in oil exploration: the case of Argentina}

\section{Q43;}

C13;

G11

\section{KEYWORDS}

Investing

in exploration;

\begin{abstract}
This article examines the cyclic behavior of investing in upstream oil and natural gas exploration in Argentina and some of the main economic variables in agreement with Kydland and Prescott's methodology (1990). The objective is to clarify the determinants involved in deciding to make said investment in a developing country. Some of the main qualitative results are related to the fact that the economic variables may not be explained individually, to the relevance of uncertainty and its impact on the agents' rationality when making decisions especially in developing countries as is the case that is analyzed. These aspects must
\end{abstract}

Correo electrónico: mrecalde@uns.edu.ar (M.Y. Recalde). 
Correlation coefficient; Determinants; Uncertainty particularly be taken into consideration when analyzing the sustainability of the energy system and the energy policy's design, mainly given the role of energy supply in socio-economic development.

(c) 2010 Asociación Cuadernos de Economía. Published by Elsevier España, S. L. All rights reserved.

\section{Introducción}

La inversión es la variable fundamental de la actividad económica. Su impacto sobre el ciclo de las empresas, la demanda agregada y el nivel de actividad global de la economía, así como su rol determinante para el proceso de acumulación nacional, la convierten en una variable crítica para el desempeño económico ${ }^{1}$.

En un sistema energético, entendido éste como un conjunto de cadenas energéticas ${ }^{2}$, la seguridad de abastecimiento se encuentra determinada por el equilibrio dinámico, a lo largo del tiempo, entre la demanda y la oferta en cada cadena energética. Dicho equilibrio depende en forma directa de la inversión coordinada, en todos los segmentos de las distintas cadenas. La inversión es entonces, en este caso, una variable esencial para la sostenibilidad del sistema. Al mismo tiempo, la importancia del abastecimiento energético para el desarrollo socioeconómico de los distintos países, y en particular para el caso de los países en desarrollo (Cleveland, 2003; Cleveland et al., 1984; Stern, 2009; Stern y Cleveland, 2004), resalta la relevancia de la inversión en los sistemas energéticos para el desarrollo de los mismos.

Para un sistema energético, los recursos primarios, recursos naturales, son los factores críticos. Así, cada sistema energético tiene una estructura diferencial atendiendo al peso relativo de las distintas fuentes primarias (carbón, gas natural, petróleo, hidro-energía, leña, geotermia, eólica, biomasa, entre otras) dentro de su oferta interna de energía. En la actualidad, las matrices energéticas primarias del mundo presentan una estructura altamente concentrada en hidrocarburos (IEA, 2007; Secretaría de Energía de la Nación, 2008; World Energy Council, 2007). Esta composición de las matrices primarias convierte a los hidrocarburos en los recursos estratégicos de los sistemas energéticos, y en muchos casos esto implica que la inversión en exploración en el upstream constituya una variable crítica para el sistema económico, al menos a corto y medio plazo (De Dicco, 2004; Kozulj y Pistonesi, 2004; Guzowski y Recalde, 2008).

1. Keynes (1936), en su desarrollo en La teoría general, le da a la decisión de inversión el rol central en la determinación de la demanda agregada en el punto de demanda efectiva, lo que en última instancia determina el nivel de producción y empleo.

2. A lo largo de este trabajo se entiende el sistema energético como: “... una sucesión de actividades, las cuales, a partir de una cierta dotación de recursos naturales, permiten satisfacer los requerimientos de energía de la sociedad, tanto los derivados del estilo de vida de la población como los asociados a la actividad económica productiva. En particular, suele definirse como un conjunto de cadenas energéticas, respondiendo cada una de ellas a una fuente determinada..." (Hasson y Pistonesi, 1988, p. 1; Bouille, 2004, p. 57).
Argentina es un país con una alta dependencia hidrocarburífera. En el año 2007, el $89 \%$ de la oferta interna de energía primaria correspondía a petróleo y gas natural, mientras que, según la información de la Compañía Administradora del Mercado Mayorista Eléctrico Sociedad Anónima (CAMMESA), en el año 2008, el $58 \%$ de la generación eléctrica fue en función de generación térmica, principalmente centrales de ciclo combinado, turbo vapor y turbinas a gas, y el $55 \%$ de la potencia instalada en el Mercado Eléctrico Mayorista (MEM) es térmica. Sin embargo, en este contexto de alta relevancia de los hidrocarburos, se ha observado en el país en los últimos años un déficit en la inversión en exploración, aspecto que, en conjunción con otros factores, ha llevado a una caída en el horizonte de vida de las reservas.

La relevancia de los recursos hidrocarburíferos para el sistema energético nacional vuelve particularmente importante el estudio de los determinantes microeconómicos de la inversión en el upstream de estos recursos. Tal como menciona Fischer (2004), la inversión en la industria de combustibles fósiles requiere del entendimiento de la dinámica de la oferta y la demanda de los mercados de petróleo y gas natural, los cuales presentan una alta ciclicidad, así como de las características particulares de la industria, y de la forma en la cual los productores de hidrocarburos realizan sus valuaciones de activos y decisiones de financiamiento. No obstante este reconocimiento en la literatura, a nivel local, la mayoría de las explicaciones a las caídas en inversión en Argentina han provenido casi exclusivamente desde el análisis de las variables económicas.

En este contexto, en este trabajo se aborda la temática de los determinantes microeconómicos de la inversión en upstream de petróleo y gas natural para el caso particular del sistema energético argentino. El objetivo primordial es discernir en qué medida los aspectos relacionados exclusivamente con los precios y la rentabilidad son suficientes para explicar la falta de inversiones en exploración en el país. Con estos fines el trabajo ha sido estructurado en tres apartados. En un primer lugar, se analiza la evolución de la inversión en el upstream en Argentina realizando un análisis en términos cualitativos, intentando aproximar relaciones de correlación entre la proxy utilizada para la inversión en exploración y algunas variables relevantes, eliminando la tendencia estocástica y manteniendo el componente cíclico mediante la aplicación del filtro de Hodrick y Prescott a las series. En segundo lugar, se aborda la temática de otros posibles determinantes de la inversión, sobre la base de otros estudios referidos a los determinantes microeconómicos de la inversión en otros sectores de la economía, desarrollando en términos teóricos su posible relevancia explicativa, presentando luego 
un esquema de análisis del comportamiento de la inversión para el upstream. Finalmente, se presentan las consideraciones finales del trabajo.

\section{Inversión y variables económicas: análisis empírico}

Existe en la literatura económica una tendencia a establecer una interrelación (positiva) predominante entre las variables económicas relacionadas con la rentabilidad y el comportamiento de la inversión. En el caso particular del sector energético argentino, diversas fuentes remarcan que han sido y son las variables relacionadas con la rentabilidad del segmento del upstream, principalmente el precio y la apropiación de la renta hidrocarburífera, las responsables del comportamiento de la inversión. En este apartado se aborda esa relación con el fin de discutir en qué medida dichas variables constituyen el principal factor explicativo de las decisiones de inversión.

Antes de avanzar en el desarrollo, es necesario resaltar que una de las principales limitaciones que se enfrenta al momento de realizar un análisis de los determinantes de la inversión en exploración se encuentra en la disponibilidad y la confiabilidad de la información. Por un lado, no existen en Argentina series confiables de inversión en exploración; por otro lado, no todas las posibles variables explicativas son observables o cuantificables. Así, el análisis exacto y la cuantificación de las relaciones entre las variables se vuelven dificultosos.

En este contexto, se realiza aquí un análisis cualitativo del comportamiento entre algunas variables seleccionadas y la inversión en exploración, con el fin de encontrar posibles patrones de comportamiento entre las mismas. Puesto que el objetivo principal del análisis es realizar una aproximación del comportamiento de las variables, se ha analizado el movimiento cíclico de las mismas, analizando los patrones de comportamiento conjunto. El fin es intentar determinar si las variables seleccionadas presentan una alta correlación con la inversión y cómo ha sido el sentido de la relación observada en el período de análisis.

\subsection{Rentabilidad y financiamiento}

En el caso particular del sector energético, entre las variables económicas más remarcadas en la literatura en torno a su impacto sobre la inversión se encuentran la rentabilidad y el financiamiento.

Por un lado, en este segmento de la cadena energética la rentabilidad se presenta como una conjunción de factores. La rentabilidad de las empresas puede analizarse en función de la ganancia obtenida de la actividad y la apropiación de un porcentaje de la renta hidrocarburífera. Asimismo, la rentabilidad propia de la actividad varía en función de una conjunción de variables que se combinan, tales como la evolución de la demanda del recurso, los precios obtenidos y la capacidad de producción de la empresa.

De acuerdo a Fischer (2004), las grandes compañías petroleras y gasíferas desean monetizar sus reservas tan pronto como sea posible con el fin de amortizar el capital invertido. Este aspecto, sin embargo, entra en contradicción con la sustentabilidad a largo plazo de la compañía, y del sector energético en su conj unto ${ }^{3}$. El autor destaca que las oportunidades de reinversión no siempre se ajustan a las tasas de explotación, con lo que la caída en la producción y en los flujos de caja es inevitable. Para evitar esta caída en los flujos de caja a raíz de la caída en la producción, las empresas deberán invertir en la exploración de nuevos pozos o adquirir áreas no desarrolladas. Fischer (2004) subraya que, en general, las grandes compañías bien capitalizadas se encuentran en condiciones de afrontar el lag entre el descubrimiento de las reservas y la puesta en producción de los pozos $^{4}$, lo que favorece la exploración; mientras que las pequeñas compañías suelen adoptar una estrategia de adquisición. La elección de una u otra forma de mantener la capacidad productiva, así como de una combinación entre ambas, determina el perfil de riesgo de la empresa.

El rol de los precios parece ser, al menos desde la perspectiva de este trabajo, un tanto más controvertido en cuanto a la relación con la inversión. Está claro que los incrementos en los precios tienen un efecto positivo sobre la inversión en exploración. No obstante, el camino puede no ser tan directo. De hecho, Fischer (2004) destaca que, dado que los inversores energéticos suelen sobre-reaccionar al precio spot, el mercado petrolero parece ser más atractivo precisamente en los momentos de precios bajos de los hidrocarburos 5 .

Por otro lado, se encuentra la importancia de las fuentes de financiamiento para la inversión. En términos generales, la cuestión del financiamiento de la inversión se haya altamente relacionada con el estudio de la incertidumbre, las expectativas y el impacto de las crisis económicas sobre el mismo (Wray y Tymoigne, 2008). La repercusión de las posibles restricciones de financiamiento externo sobre las inversiones realizadas en cada sector depende principalmente de la forma en la cual las empresas que se desempeñan en el mismo financian sus inversiones. De acuerdo a Eichner (1976) y Lavoie (1992), uno de las principales rasgos de las grandes empresas económicas que se mueven en mercados con características oligopólicas, a las que los autores Ilaman megacorps y que son altamente similares a las empresas petroleras, es el hecho de que suelen financiar sus inversiones con fondos propios, en lugar de utilizar financiamiento externo (Recalde, 2010).

En este sentido, de acuerdo con la IEA (2009), históricamente las inversiones en el upstream de hidrocarburos han estado menos influenciadas por los cambios en el ambiente

\footnotetext{
3. Esto es así porque el incremento en la tasa de explotación del recurso, al tratarse de un recurso no renovable, disminuye la disponibilidad esperada del mismo en un futuro. De esta forma, un incremento en la tasa de extracción del recurso, sin aumentos en la inversión en nueva exploración y en técnicas de recuperación secundaria y terciaria, implica necesariamente una reducción en la disponibilidad del mismo y una caída en el horizonte de reservas, atentando contra la sustentabilidad futura de la empresa (para la cual las reservas hidrocarburíferas constituyen los activos principales) y del país (que requiere del recurso para el abastecimiento energético).

4. En general, este lag oscila entre cuatro y siete años tendiendo a las características propias del yacimiento.

5. Tal como remarca el autor, los precios bajos Ilevan a períodos de subinversión, lo que conduce a períodos de desabastecimiento, seguidos por aumentos de precios y sobreinversión, produciendo nuevas caídas en los precios.
} 
de créditos, puesto que dichas inversiones recaen en forma más directa sobre el financiamiento propio, en lugar de financiamiento externo. Este tipo de actividades suelen estar caracterizadas por un alto nivel de autofinanciamiento, a partir de su propio flujo de caja, y bajos niveles de debtequity ratios, aspecto que se condice con el análisis de las empresas en el marco de la megacorp poskeynesiana. De esta forma, los impactos de la crisis financiera, es decir, los recortes en el financiamiento proveniente de los mercados financieros mundiales, parecerían no ser un impedimento para llevar a cabo los proyectos de inversión. No obstante, la IEA (2009) reconoce que, por la situación del mercado financiero durante los años 2007 y 2008, como consecuencia de la crisis financiera global, las principales empresas petroleras del mundo han debido recurrir al mercado financiero para alcanzar sus metas de inversión, dada la reticencia de las mismas a reducir los dividendos repartidos, aspecto que estaría revirtiendo la tendencia al financiamiento de la inversión en el sector.

\subsection{Características de la inversión en exploración}

La actividad del upstream de hidrocarburos es una actividad altamente capital intensiva, que requiere grandes inversiones en capital físico, caracterizada por un alto riesgo minero propio del negocio (Caruso, 2003; Gadano, 1998). A lo largo de la actividad del upstream, se desarroIlan tres etapas: exploración, desarrollo y extracción, o producción. Cada una de estas etapas tiene asociada una categoría de inversión en particular, con un peso diferente en el costo total de inversión de la actividad. La etapa de exploración es la que tiene menor participación en la inversión, solamente el $15 \%$ aunque es la etapa que presenta el mayor nivel de riesgo determinado no sólo por el riesgo propio del negocio, sino también por el riesgo minero propio de la actividad. Dicha actividad depende de las condiciones técnicas y geológicas del área, y los factores económicos, y tiene como objetivo principal detectar la acumulación de recursos hidrocarburíferos en un determinado yacimiento, identificándolos por su grado de certidumbre (conocimiento geológico y geofísico acerca de la existencia de calidad y magnitud de los depósitos) y su factibilidad económica de recuperación, para luego finalmente descubrir los hidrocarburos que se encuentren en condiciones técnico-económicas de ser explotadas (IDEE/ FB, 2006). Del total de esa inversión, el $70 \%$ de los costos corresponde a la perforación de pozos, siendo el 30\%restante correspondiente a la realización de prospectivas geológica y geofísica. Por su parte, la etapa de desarrollo representa el $50 \%$ de la inversión, la cual se divide entre la inversión en las instalaciones requeridas para la producción y el transporte y la perforación de pozos de desarrollo. Finalmente, la etapa de producción representa el $35 \%$ del costo de inversión.

Es importante resaltar que, dentro de estas distintas partidas de inversiones, las realizadas en la etapa de exploración son particularmente importantes al momento de otorgar sustentabilidad en el tiempo al sistema, pues son las encargadas de extender el horizonte de vida de las reservas para un determinado ritmo de explotación. Al mismo tiempo, dado el riesgo minero propio de la actividad, estas inversiones son las que presentan mayor riesgo, y es justa- mente la existencia de riesgo minero lo que hace que exista incertidumbre en torno a la clasificación de las reservas, aun en aquellas reservas descubiertas ${ }^{6}$.

\subsubsection{Metodología}

Motivado por la cada vez más frecuente existencia de crisis económicas y contracciones internacionales, con impacto diferencial en las distintas economías del mundo, se ha reflotado con mayor intensidad el análisis empírico del comportamiento de las variables a lo largo del ciclo económico. Así, la caracterización estadística de dicho comportamiento se ha convertido en una rama de análisis fuertemente difundida en las últimas décadas. Usualmente esta literatura se concentra en analizar los hechos estilizados para diferentes países y momentos del tiempo (Feal Zubimendi et al., 2010; Kydland y Prescott, 1990; Kydland y Zarazaga, 1997), tratando de identificar co-movimientos o movimientos conjuntos entre las variables. Estos movimientos conjuntos, usualmente de alguna variable económica (real o monetaria) y el PBI, se estudian mediante la medición del coeficiente de correlación cruzado entre las mismas.

Para realizar estos estudios, es necesario utilizar algún procedimiento con el fin de eliminar la tendencia estocástica (no estacionaria), manteniendo el componente cíclico (estacionario), aplicando filtros a las series. Uno de los principales motivos por los cuales es necesario realizar este proceso de filtrado se encuentra en que, en general, la mayoría de las variables macroeconómicas son no estacionarias (André et al., 2002). En la actualidad, existen diferentes metodologías que pueden ser utilizadas para separar el componente cíclico del tendencial, muchas de las cuales han surgido a partir del trabajo de Kinland y Prescot (1982) (Feal Zubimendi et al., 2010).

A pesar de haber surgido principalmente para el análisis de los ciclos económicos y de los hechos estilizados, y si bien en su mayoría este análisis no ha sido empleado en estudios de las características aquí propuestas, se considera que esta metodología puede ser de mucha utilidad para analizar el comportamiento de la inversión en el sector energético.

En este contexto, el motivo principal por el cual se ha optado por utilizar esta metodología radica en que el fin es observar en qué forma se ha dado el movimiento conjunto de las variables analizadas en el período de referencia. No obstante, es importante destacar en esta instancia que el uso de este tipo de coeficiente de correlación para el análisis de la inversión no permite obtener resultados suficientemente concluyentes. Posiblemente en forma alternativa sería enriquecedor utilizar un análisis de regresión, mediante técnicas de datos de panel, e incluso empleando otras variables explicativas, así como alguna variable dependiente de características monetarias. Sin embargo, un estudio de datos de panel requeriría información desagregada de empresas de niveles de inversión, rentabilidad, entre otras, información que no se encuentra disponible en el país.

6. Usualmente el estudio de la clasificación de reservas se realiza en forma simple recurriendo a la matriz de McKelvey, Ilamada así por el geólogo Estadounidense Vincent McKelvey. Se trata de un esquema bi-dimensional que combina el criterio de seguridad geológica con la factibilidad económica (Cleveland y Morris, 2006). 


\subsubsection{Descripción de las técnicas de filtrado ${ }^{7}$}

Tal como se mencionó anteriormente, el análisis de los co-movimientos o movimientos conj untos entre las variables a lo largo de los ciclos económicos requiere de una aplicación previa de técnicas de filtrado, las cuales permiten obtener el comportamiento cíclico de las variables bajo estudio. De Lucas Santos et al. (2009) destacan que ha sido precisamente la obtención del componente cíclico mediante las técnicas de filtrado utilizadas el aspecto más controvertido en esta literatura. Todos los mecanismos de filtrado existentes poseen algún tipo de elemento arbitrario en su procedimiento, aspecto que dificulta llegar a un resultado adecuado y que hace que la elección de uno u otro elemento condicione necesariamente los resultados obtenidos.

De Lucas Santos et al. (2009) argumentan que, en última instancia, los distintos filtros existentes se consideran aproximaciones a los filtros ideales ${ }^{8}$. De acuerdo con los autores, tres son los filtros más representativos de la literatura. En primer lugar, el filtro de Hodrick y Prescott (1980) es un filtro básico en su versión de filtro de paso alto, pues elimina la tendencia de las series desestacionalizadas. En segundo lugar, el filtro de Baxter y King (1995) es un filtro de medias móviles simétrico en su versión de paso banda. Y, finalmente, los filtros de la familia Butterworth, también en una versión paso banda, son filtros con estructuras ARMA. No obstante, aun cuando los tres filtros cumplan a grandes rasgos las características principales de un filtro ideal, incluso este último puede tomar una estructura que induzca a ciclos espurios. Esto se debe en cierta medida al hecho de que un filtro es considerado óptimo cuando, al filtrar la serie, el residuo es ruido blanco, algo que no se verifica en la gran mayoría de las series económicas ${ }^{9}$ (De Lucas Santos et al., 2009).

Así, los distintos trabaj os empíricos no han logrado aún ser concluyentes en torno a qué filtro debe ser más correctamente utilizado en la práctica, y en general se trata de elecciones de los autores, basadas en aspectos relacionados con la simplicidad de cada filtro, o con la literatura preexistente en el estudio de cada caso. Por estos motivos, en este trabajo se ha decidido seguir la metodología empleada por estudios precedentes para el comportamiento de los ciclos en Argentina. En este sentido, siguiendo a Feal Zubimendi et al. (2010), se utiliza aquí el filtro de Hodrick y Prescott (HP) para la descomposición del componente cíclico y tendencial de las series.

El filtro HP consiste en la extracción de una tendencia estocástica que se mueve suavemente a lo largo del tiempo y no se encuentra correlacionada con el componente cíclico. El método propone que el componente tendencia de una serie es la que minimiza:

7. Para un análisis más detallado de las características de las técnicas de filtrado y su comparación en la obtención de resultados se recomienda ver De Lucas Santos et al. (2009).

8. Entre las propiedades que debe cumplir un filtro ideal destaca que sea operativo, que extraiga el rango específico de periodicidades que se desea dejando inalteradas las propiedades del componente extraído, que sea simétrico, que produzca series estacionarias incluso cuando se aplique a datos con tendencia y que no dependa del tamaño (De Lucas Santos et al., 2009).

9. Véase, por ejemplo, De Lucas Santos et al. (2009), figura 3, para una demostración de este aspecto.
$\sum_{t=1}^{T}\left(y_{t}-\tau_{t}\right)^{2}+\lambda \Sigma_{t=3}^{T}\left(\Delta^{2} \tau_{t}\right)^{2}$

Donde:

$\Delta^{2}=(1-L)^{2}$

L: operador de rezagos

$\tau_{\mathrm{t}}$ : componente de tendencia para la serie

$\lambda$ : parámetro que penaliza la variabilidad de la tendencia en función de la frecuencia de la serie bajo estudio ${ }^{10}$.

Para realizar la interpretación de los resultados del movimiento conjunto entre las variables, $X_{t+i}$ es necesario analizar la magnitud y el signo del coeficiente de correlación cruzada $\rho(j), j \in\{0, \pm 1, \pm 2, \pm 3, \pm 4, \pm 5\}$ en su mayor valor.

En primer lugar, la intensidad de la relación entre las variables se determina analizando el valor absoluto del mayor coeficiente de correlación cruzado, presentando una relación fuerte si $0,5 \leq|\rho(j)| \leq 1$, siendo la relación débil si $0,2 \leq|\rho(\mathrm{j})| \leq 0,5$, y no estableciéndose relación (variable acíclica) si $|\rho(j)| \leq 0,2$. En segundo lugar, se puede observar la fase de desplazamiento de cada una de las series. Si el $\rho(j)$ alcanza su mayor valor para $\mathrm{j}<0$, la variable anticipa el comportamiento de la variable de referencia (leading); si el $\rho(j)$ alcanza su mayor valor para $\mathrm{j}=0$, las variables son contemporáneas, es decir, se mueven en el mismo momento del tiempo; mientras que, si el mayor valor del $\rho(j)$ se alcanza para $\mathrm{j}>0$, la variable sigue a la variable de referencia o se encuentra rezagada (lagging). Finalmente, es posible establecer la dirección del comportamiento de las variables de forma tal que, si el valor más alto del coeficiente de correlación cruzado es positivo, la relación entre las variables es directa (procíclica); si es negativo, la relación es indirecta (contracíclica), y si el valor es cercano a cero, es nula (acíclica).

\subsubsection{Datos e información utilizada}

Sobre la base teórica introducida en la sección anterior, el estudio se lleva a cabo para las series anuales de las siguientes variables ${ }^{11}$ :

- I: inversión en exploración. Número de pozos exploratorios totales perforados por año.

- $I_{\text {petr: }}$ inversión en exploración de petróleo. Número de pozos exploratorios petroleros perforados por año.

- $I_{G N}$ inversión en exploración de gas natural. Número de pozos exploratorios de gas natural perforados por año.

- $\mathrm{PP}_{\mathrm{Argen}}$ : precio promedio del petróleo en boca de pozo en Argentina. Precio promedio del crudo de venta al mercado interno y externo por provincia, ponderado por volumen de venta (US\$/ $\left.\mathrm{m}^{3}\right)$.

- $\mathrm{PGN}_{\text {Argen }}$ : precio promedio del gas natural en boca de pozo en Argentina. Precio promedio del gas natural en boca de pozo de venta al mercado interno y externo por provincia, ponderado por volumen de venta (US\$/ $\left.\mathrm{m}^{3}\right)$.

10. Aquí, siguiendo el trabajo de Feal Zubimendi et al. (2010), y dado que las series utilizadas son anuales, se emplea un valor de $\lambda=100$.

11. No se incluyen en este análisis las variables relacionadas con el financiamiento de la inversión en las empresas petroleras en Argentina, puesto que no es información que se encuentre disponible en series factibles de ser utilizadas en el análisis de la correlación. 
- $\mathrm{PP}_{\mathrm{WTI}}$ : precio spot WTI. Precios internacionales para el crudo de referencia en Argentina, es decir, el WTI, de la British Petroleum Statistical Report (2009) (US\$/ BII).

- RConjunta: renta conjunta. Monto total de la renta hidrocarburífera total apropiada por los productores hidrocarburíferos.

- PRenta: participación en la renta. Participación en la apropiación de la renta hidrocarburífera total por parte de los productores. Esta corresponde a un análisis realizado en torno a la distribución primaria de la renta, la cual se refiere a su distribución dentro del sistema productivo en general, haciendo atención a la participación de cada uno de los agentes de alguna forma dentro del proceso productivo. En el caso de Argentina, en general esta distribución le corresponde al Estado Nacional o Provincial, como dueño del recurso (teniendo en cuenta la regulación vigente); las empresas productoras, en el caso argentino todas ellas empresas privadas, como agentes del upstream, y los consumidores o compradores del recurso (\%).

- TCE: tasa de crecimiento económico. Tasa de crecimiento anual esperada para el período siguiente en la economía.

El análisis de la correlación entre $\mathrm{PGN}_{\text {Argen }}, \mathrm{PP}_{\text {Argen }}, \mathrm{I}_{\text {Petr }}$ e $\mathrm{I}_{\mathrm{GN}}$ se realiza para el período 1993-2003, puesto que solamente hasta dicho año se cuenta con información desglosada. Se reconoce aquí la posible limitación del análisis dada la reducción de los datos ${ }^{12}$; no obstante, los resultados se presentan a título ilustrativo.

Las fuentes de información utilizadas para el análisis han sido el Instituto Nacional de Estadísticas y Censos (INDEC), la Secretaría de Energía de la Nación, en función de información provista por las empresas, complementada con datos de IDEE/ FB, el Instituto Argentino de la Energía (IAE) e información de los balances de las empresas líderes del sector hidrocarburífero en el país en el período bajo análisis ${ }^{13}$. Los datos de la renta hidrocarburífera conj unta y de la participación de la misma se basan en cálculos propios realizados según información de diferentes fuentes estadísticas y en torno a la legislación nacional existente que establece la distribución de la renta ${ }^{14}$ entre los distintos agentes operantes en el mercado hidrocarburífero argentino ${ }^{15}$. La metodología empleada para el cálculo se presenta en el anexo al final del trabajo.

Tal como se ha mencionado, dado que la información oficial disponible respecto a la inversión exploratoria es la relacionada con el número de pozos perforados por año, esta ha sido la variable utilizada como proxy de la inversión en exploración en el segmento. En cierta forma, esta variable parece ser una buena proxy de la inversión en exploración puesto que, tal como se ha mencionado, la perforación de pozos representa el $70 \%$ de la inversión en exploración. Sin

12. En general, para el empleo de la metodología del filtro seleccionado se requiere un $t>15$.

13. Particularmente el caso de REPSOL YPF, empresa líder del oligopolio energético nacional, dada su participación en el mercado de gas natural y petróleo crudo.

14. Para el cálculo de la renta se ha basado en el concepto teórico de la Renta Absoluta de Monopolio de Marx (1894), siguiendo, entre otros autores, a Mansilla (2007).

15. Para una descripción de la metodología y los resultados obtenidos en la estimación de la evolución de la renta se recomienda ver Recalde (2010). embargo, se reconoce aquí que la aproximación de la actividad exploratoria únicamente a partir de la perforación de pozos es incompleta, particularmente dados los progresos tecnológicos en la realización de prospectivas que otorga más precisión disminuyendo la demanda de perforación con el fin de indagar sobre la existencia del recurso, lo que puede explicar la caída en la perforación de pozos. No obstante, la inexistencia de información monetaria confiable en términos de inversión monetaria impide realizar cualquier otro tipo de análisis.

\subsubsection{Resultados obtenidos}

Antes de avanzar en el análisis de los resultados obtenidos, se considera útil analizar el comportamiento que ha tenido la proxy de la inversión en el período bajo estudio. La figura 1 muestra la evolución de la actividad exploratoria desde 1973 a 2007 en Argentina. La misma presenta un comportamiento oscilante con cinco máximos relativos (1973, 1977, 1985, 1995 y 2006) y dos mínimos relativos en 1992 y 2002. La participación de la actividad de perforación dentro del total de perforación es bajo, inferior al $25 \%$ en todos los casos salvo al inicio de la serie. Se observa además una caída de la actividad desde el año 1995 hasta 2002, una recuperación hacia el año 2006, aunque sin lograr los niveles anteriores, y una nueva caída en 2007.

En la figura 2 se muestran la descomposición de los ciclos y las tenencias para cada una de las series analizadas.

Una vez descompuestas cada una de las series, se realizó el análisis de las funciones de correlación cruzadas, con el fin de estudiar la intensidad, la dirección y la fase de desplazamiento de cada una de las variables seleccionadas en relación con la variable utilizada como proxy de la inversión. En la tabla 1 se presentan los resultados obtenidos de los coeficientes de correlación cruzados entre el componente cíclico de las variables seleccionadas, una vez aplicado el filtro HP.

Tal como se desprende de los resultados, el comportamiento entre la inversión en exploración y las variables seleccionadas en el período bajo análisis ha sido muy errático, no presentándose prácticamente patrones de relación marcados.

Por un lado, la mayoría de las variables presentan una intensidad de movimiento débil, ya que su coeficiente de correlación es inferior o ínfimamente superior a 0,5

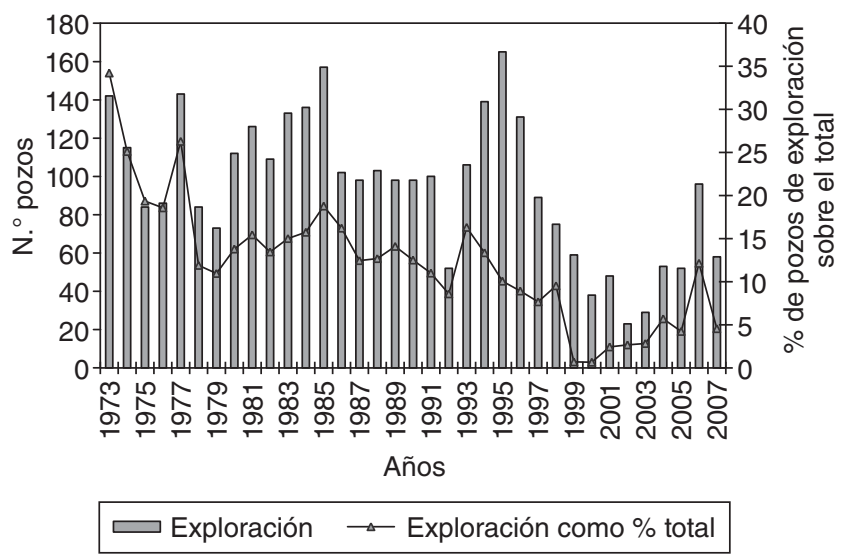

Figura 1 Evolución de los pozos perforados. 1973-2007. Fuente: Elaboración propia en función de información SE, IAE y FB. 


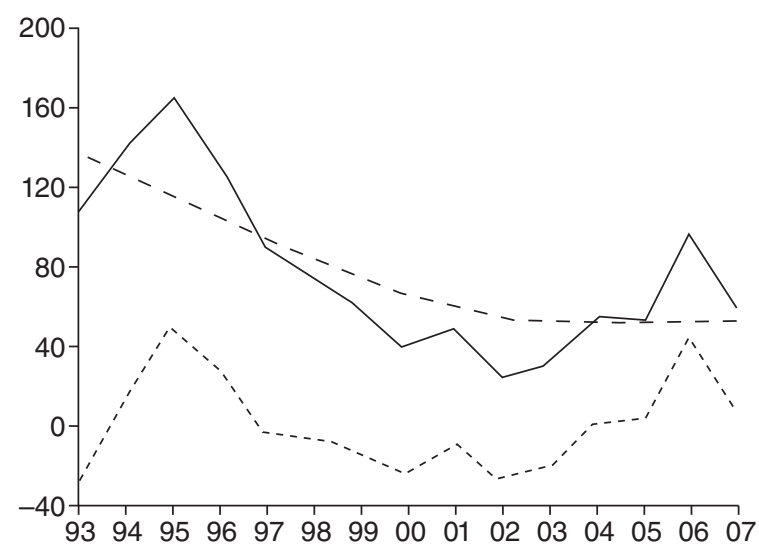

- I -.- ICICLO - - ITEND
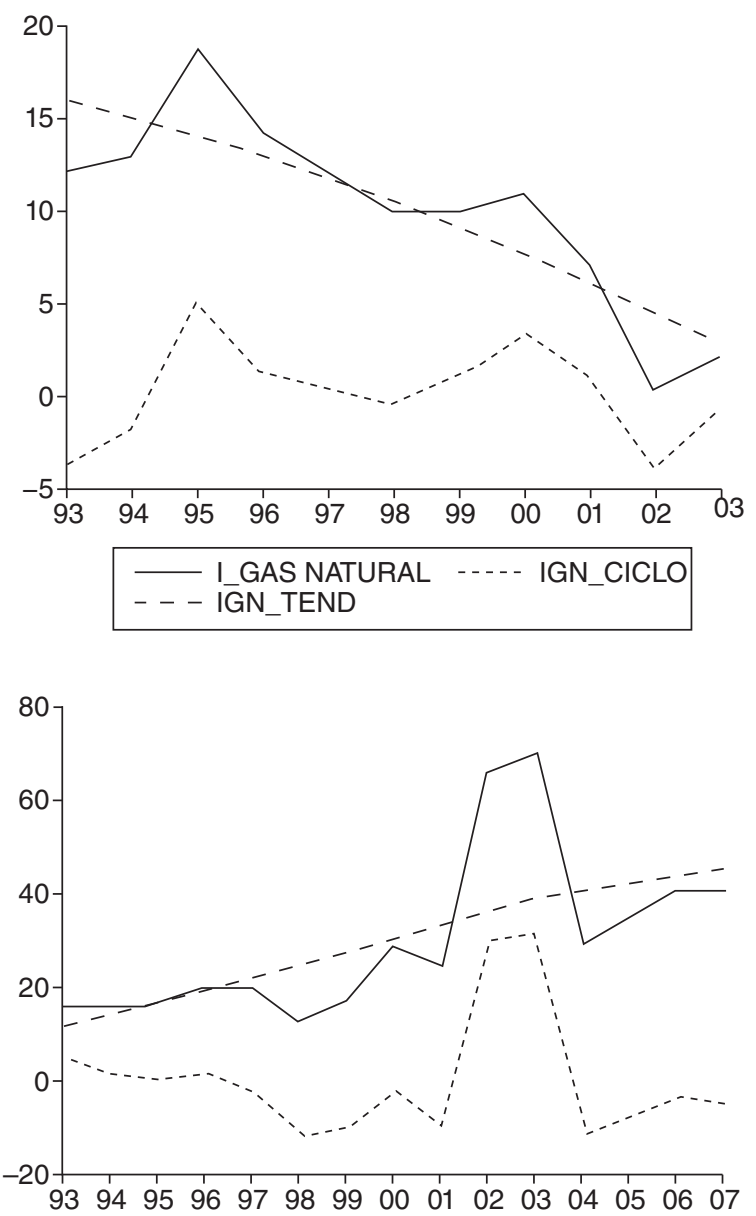

$$
\begin{aligned}
& \text { - PPARGEN - - - PPARGCICLO } \\
& \text { - - PPARGTEND }
\end{aligned}
$$
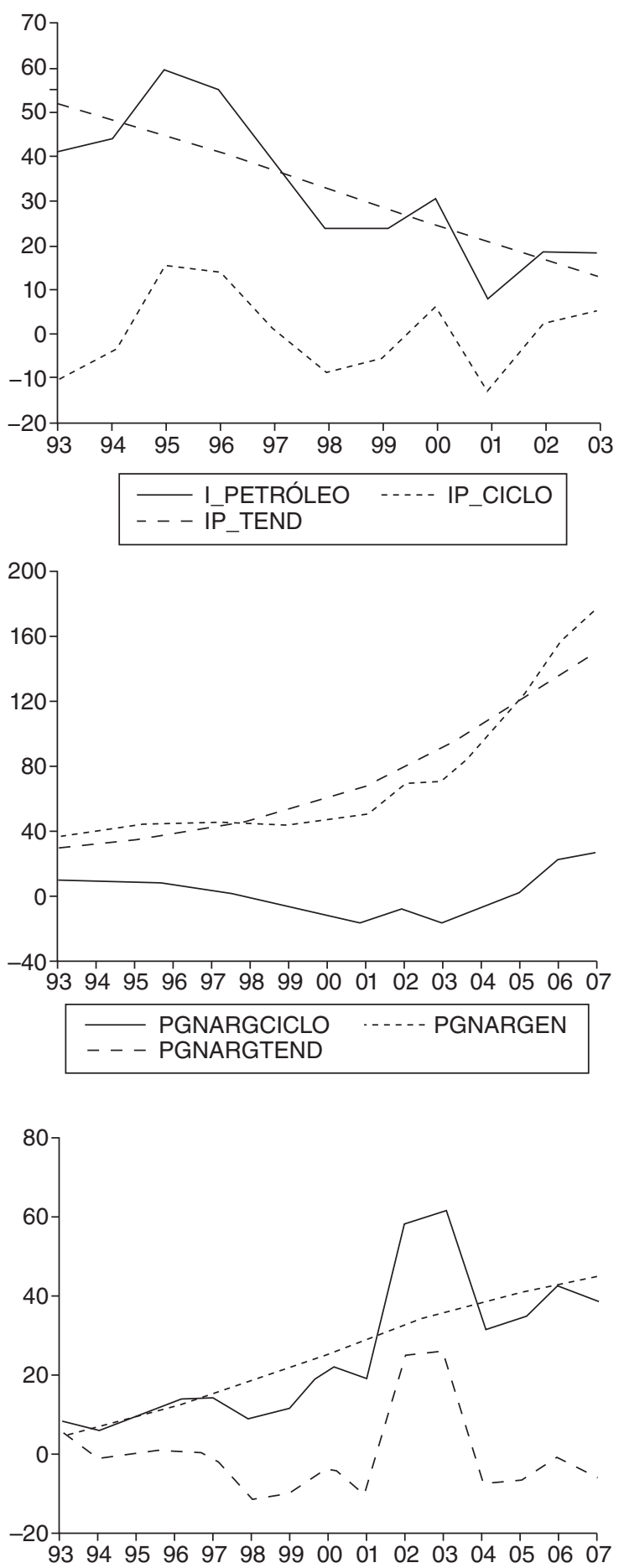

\begin{tabular}{ll}
\hline- RCONJUNTA & -..- RCONJTEND \\
\hline-- RCONJCICLO &
\end{tabular}

Figura 2 Descomposición de las series utilizando el filtro HP. Fuente: Elaboración propia. Las variables presentadas en los gráficos se describen como: I: I; ICICLO: componente cíclico de I; ITEND: componente tendencial de I;I_PETROLEO: I Ietr; IP_CICLO: componente cíclico de $I_{\text {Petr }}$; IP_TEND: componente tendencial de $I_{\text {Petr }} ; I_{-}$GASNATURAL: I IN; IGN_CICLO: componente cíclico de I IN; IGN_TEND: componente tendencial de $I_{G N} ;$ PGNARGEN: PGN ${ }_{\text {Argen }}$; PGNARGCICLO: componente cíclico del PGN ${ }_{\text {Argen }}$; PGNARGTEND: componente tendencial del PGN ${ }_{\text {Argen }}$; PPARGEN: $\mathrm{PP}_{\text {Argen; }}$ PPARGCICLO: componente cíclico del PP Argen; PPARGTEND: componente tendencial del PP Argen; RCONJ UNTA: $\mathrm{R}_{\text {conjunta; }}$ RCONJ CICLO: Componente cíclico de $\mathrm{R}_{\text {conjunta; }}$ RCONJ TEND: componente cíclico de $\mathrm{R}_{\text {conjunta. }}$. 
Tabla 1 Coeficientes de correlación para las variables

\begin{tabular}{|c|c|c|c|c|c|c|c|c|c|c|c|}
\hline \multirow[t]{2}{*}{ Variables } & \multicolumn{11}{|c|}{ Coeficientes de correlación } \\
\hline & -5 & -4 & -3 & -2 & -1 & 0 & 1 & 2 & 3 & 4 & 5 \\
\hline \multicolumn{12}{|c|}{ Inversión en pozos exploratorios totales } \\
\hline RConj unta & $-0,0020$ & 0,1224 & 0,1467 & 0,2610 & $-0,0092$ & $-0,2946$ & $-0,0905$ & $-0,1590$ & $-0,4239$ & $-0,2894$ & $-0,0898$ \\
\hline PRenta & 0,0917 & 0,1266 & 0,1033 & 0,0777 & $-0,2578$ & $-0,5016$ & $-0,2404$ & $-0,2382$ & $-0,3322$ & $-0,1944$ & $-0,0473$ \\
\hline Ganancia & $-0,1190$ & $-0,1191$ & $-0,1700$ & $-0,1588$ & $-0,1878$ & 0,4858 & 0,4168 & 0,3204 & 0,2562 & 0,0861 & $-0,044$ \\
\hline TCE & $-0,0939$ & $-0,0600$ & $-0,0277$ & 0,0929 & 0,4119 & 0,2187 & 0,2171 & 0,3608 & 0,0827 & $-0,0937$ & $-0,1231$ \\
\hline \multicolumn{12}{|c|}{ Inversión en pozos exploratorios petroleros } \\
\hline $\mathrm{PP}_{\text {Argen }}$ & $-0,0706$ & $-0,0408$ & 0,0564 & 0,2207 & 0,1080 & 0,4313 & $-0,0219$ & $-0,0744$ & $-0,3479$ & $-0,1004$ & 0,1187 \\
\hline $\mathrm{PP}_{\mathrm{WTI}}$ & 0,0687 & 0,1242 & $-0,0570$ & $-0,2483$ & $-0,3231$ & 0,2894 & $-0,0134$ & $-0,3674$ & $-0,2675$ & 0,0151 & 0,1587 \\
\hline \multicolumn{12}{|c|}{ Inversión en pozos exploratorios gas natural } \\
\hline $\mathrm{PGN}_{\text {Argen }}$ & $-0,0035$ & 0,0095 & 0,0036 & $-0,0621$ & 0,0939 & $-0,4703$ & $-0,1681$ & $-0,1635$ & $-0,2006$ & $-0,1572$ & 0,0791 \\
\hline
\end{tabular}

$(0,5 \leq|\rho(j)| \leq 1)$. Solamente $\rho(j)$ para la relación entre la inversión en pozos exploratorios totales y la participación en la renta conjunta se presenta un valor levemente superior a 0,5. No obstante, se observa que, en el período de análisis, ambas variables han sido contemporáneas y la relación que se ha presentado es inversa (contracíclica), contrariamente a lo que se esperaría.

En cuanto a la relación entre la inversión en exploración y los precios, se observa, en primer lugar, que la intensidad de la relación es débil en todos los casos. En los precios promedios de los recursos en Argentina, las variables parecen ser contemporáneas, aunque no es posible establecer una conclusión en torno a si la relación entre los precios y la inversión ha sido directa o inversa, pues para el caso del petróleo parece haber sido directa, mientras que para el gas natural es inversa. En este sentido, no es posible concluir si la relación planteada por Fischer (2004) en torno a la relación entre los precios spot del WTI y la inversión en exploración se reproduce en Argentina o no.

Finalmente, las dos variables que presentan el signo y el desplazamiento de fase esperado, aunque no una relevante intensidad de movimiento, son la ganancia y la tasa de crecimiento de la economía. Ambas variables presentan una relación directa con la perforación de pozos, siendo la ganancia contemporánea, y liderando la fase la tasa de crecimiento de la economía. Este resultado se condice con lo esperado, puesto que, desde el punto de vista de los incentivos privados a la inversión, una economía con altas tasas de crecimiento y elevados niveles de ganancia para el sector en cuestión genera expectativas positivas a la inversión en el sector. Más aún, tal como reafirman diferentes estudios empíricos, existe una alta interrelación y causalidad entre el consumo de energía y el crecimiento económico (Asafu-Adjaye, 2000; Oh y Lee, 2004; Lee, 2005; Soytas y Sari, 2003; Francis et al., 2007; Zachariadis, 2007; Lee y Chang, 2007; Sari y Soytas, 2007; Narayan y Smyth, 2009), aspecto que convierte la tasa de crecimiento esperado de la economía en una buena proxy de la tasa de crecimiento esperada de la demanda.
Si bien se reconoce que los resultados no presentan un alto valor explicativo, el objetivo de este apartado ha sido observar el comportamiento cíclico que ha tenido la perforación de pozos con respecto a las variables relacionadas con la rentabilidad de la economía y la tasa de crecimiento del país, como un posible indicador del desempeño de la economía y las posibles expectativas futuras. Los resultados obtenidos avalan en cierta forma la hipótesis en torno a la debilidad de la capacidad explicativa que, en forma aislada, tienen la evolución de los precios internos e internacionales en Argentina, así como la evolución de la renta hidrocarburífera apropiada para explicar la caída en la inversión en exploración en los últimos decenios. No se ha encontrado una intensidad de movimiento fuerte para ninguna de las variables económicas utilizadas en el período de análisis. Por estos motivos, en la siguiente sección se avanza en la búsqueda de otro tipo de determinantes.

\section{Aproximación a otros determinantes de la inversión}

Ante los resultados encontrados en la sección anterior, aun reconociendo las debilidades del análisis principalmente relacionadas con la falta de disponibilidad de información, se abordan en este apartado otros factores que, desde el punto de vista de este trabajo, pueden explicar el comportamiento de la inversión en exploración principalmente en países en desarrollo como Argentina. El objetivo principal de este apartado es colaborar en la construcción de un marco analítico para el estudio futuro, de modo que se complemente el análisis de las variables económicas.

\subsection{Racionalidad, incertidumbre e inversión}

La incertidumbre es una característica fundamental de los sistemas económicos. Uno de los principales canales por los cuales la incertidumbre afecta a la actividad económica es por medio de su impacto en las decisiones de inversión. 
Los agentes económicos, al momento de la toma de las decisiones de inversión, se enfrentan a un contexto marcado por la incertidumbre. La diferente reacción ante esta situación determinará su decisión final. Así, la relación se invierte y el impacto de la incertidumbre del contexto económico es fundamental para comprender el proceso de inversión de los agentes ${ }^{16}$.

El estudio de la incertidumbre y la inversión se entrecruza con el estudio de la racionalidad de los agentes. Así, el resultado de la incertidumbre sobre la actividad económica en los análisis económicos y sus resultados dependerá de la respuesta de los individuos a dicha situación de incertidumbre (Coddington, 1982, citado en Lavoie, 1992) ${ }^{17}$.

Recientemente la literatura ha avanzado en el estudio del rol de la incertidumbre en las diversas actividades económicas, siendo el tratamiento de la relación entre la incertidumbre y la inversión en diferentes industrias y contextos económicos uno de los aspectos más estudiados (Driver y Moreton, 1991; Dixit y Pindyck, 1994; Serven, 1998; Boyle y Guthrie, 2003; Kosacoff y Ramos, 2006; Bomtempi et al., 2010; Mohn y Misund, 2009). El reconocimiento de la existencia de un contexto de incertidumbre en el cual los agentes realizan sus decisiones de inversión cambia significativamente los resultados del análisis. En efecto, según Dixit y Pindyck (1994), algunos de los principales problemas empíricos de la teoría al predecir una alta significatividad de la tasa de interés como variable explicativa de la inversión se deben, en parte, a que la misma no tiene en cuenta el efecto del contexto de incertidumbre sobre el valor futuro de la inversión ${ }^{18}$.

Aún bajo el reconocimiento de la existencia de una relación entre incertidumbre e inversión, la teoría económica no parece haber logrado, al momento, una conclusión respecto del signo de la misma. No obstante, en el campo de análisis empírico, el desarrollo de técnicas modernas para

16. Tal como lo analiza Keynes (1936), la decisión de inversión es un proceso que implica dos momentos: un primer momento de "preferencia temporal" y un segundo momento en el que entra en juego la "preferencia por la liquidez", donde el agente decide entre los dos tipos de activos en los cuales puede invertir.

17. El supuesto de que los agentes económicos se mueven con cierta racionalidad es fundamental para Ilevar adelante estudios en el ámbito de las ciencias sociales (Caldwell, 1989, citado en Lavoie, 1992). En el contexto de este análisis, es de gran aplicabilidad el concepto de racionalidad de procedimiento o racionalidad acota$\mathrm{da}$, de acuerdo a la cual las decisiones de los individuos se basan en costumbres, reglas de comportamiento y normas existentes para la toma de decisiones futuras. La racionalidad acotada con la que se guían los agentes al momento de decidir vuelve fundamentales el marco institucional y la reacción de los otros agentes que se desempeñan en la industria.

18. Dicen los autores: "Recientes estudios ha mostrado que el costo de oportunidad de la inversión puede ser alto, y que las reglas de inversión cuando lo ignoran cometen un grave error. También, este costo de oportunidad es altamente sensible a la incertidumbre respecto del valor futuro de un proyecto, de forma que los cambios en las condiciones económicas que afectan la percepción del riesgo de los fluj os futuros pueden tener un impacto importante sobre el gasto en inversión, mayor aun que un cambio en la tasa de interés. Esto puede ayudar a explicar por qué la teoría de la inversión neoclásica ha fallado tanto en proveer buenos modelos empíricos del comportamiento de la inversión, y ha llevado a pronósticos demasiado optimistas sobre la efectividad de la tasa de interés y las políticas impositivas para estimular la inversión" (Dixit y Pindyck, 1994, p. 6). analizar datos de panel ha impulsado estudios empíricos, la mayoría de los cuales soportan la hipótesis de una relación negativa (Mohn y Misund, 2009) ${ }^{19}$.

Si bien esta hipótesis de una relación negativa entre ambas variables es soportada en el presente análisis, se considera que diversos factores deben ser tenidos en cuenta, tales como las características particulares de la industria, el tipo de inversión, el contexto del país y el tipo específico de incertidumbre a la que se está haciendo referencia.

En principio, las características propias de cada industria juegan un rol fundamental. Dos aspectos de vital importancia se relacionan con el grado de competencia dentro de la industria y con el grado de irreversibilidad del capital invertido. En el primer caso, el impacto de la incertidumbre sobre la inversión suele ser negativo y de gran intensidad en industrias con alto grado de concentración. La situación, en cambio, puede ser diferente respecto al caso competitivo, tal como coinciden Hartman (1972) y Abel (1985), para quienes, en contextos de competencia perfecta, las firmas neutrales al riesgo deciden incrementar la inversión como resultado de un aumento futuro en sus ingresos. Caballero (1991), por ej emplo, destaca que los supuestos de competencia perfecta y rendimientos constantes a escala son fundamentales para obtener dicho resultado (Bond et al., 2005) ${ }^{20}$. En el segundo caso, de acuerdo con la teoría de las opciones reales de Dixit y Pindynk (1994), es la irreversibilidad del capital, juntamente con la incertidumbre y la posibilidad de posponer la decisión de inversión hasta tanto la información del futuro mejore las expectativas, lo que generalmente opera a favor de una relación negativa entre incertidumbre e inversión. De esta forma, en aquellos sectores en los cuales la inversión tiene altos componentes de costos hundidos, la incertidumbre impacta con más fuerza en forma negativa sobre la inversión.

Por otro lado, el rol de la incertidumbre en la decisión de inversión se encuentra directamente relacionado con características estructurales de los países. Los países de menor grado de desarrollo se caracterizan por presentar debilidades institucionales, al tiempo que son más proclives a las crisis económicas y políticas, y más sensibles a las crisis internacionales. Todos estos aspectos otorgan un grado de incertidumbre adicional al contexto económico en el cual ha de desarrollarse la inversión.

En el caso particular de Argentina, Kosacoff y Ramos (2006) destacan que la inestabilidad y la elevada incerti-

19. Algunos de los autores: Ogawa y Suzuki (2000) para J apón; Butzen et al. (2002) y Fuss y Vermeulen (2004) para Bélgica; Guiso y Parigi (1999) y Bontempi et al. (2009) para Italia; Sternken et al. (2002) para Holanda, y Bond y Van Reenen (2001) y Bond et al. (2005) para Reino Unido.

20. En cuanto a la evidencia empírica, Bond et al. (2005) utilizan un panel de 655 empresas de Reino Unido para el período 1987-2000, para el cual testean cuatro medidas diferentes de incertidumbre. Los autores encuentran que la incertidumbre creciente reduce la inversión en el corto plazo, y que una tendencia creciente en la incertidumbre está asociada a una menor acumulación del capital en el largo plazo. Por su parte, Bontempi et al. (2009) realizan un estudio de panel para un conjunto de firmas italianas en el período 1996-2004, con el objetivo de testear los determinantes microeconómicos de la inversión, encontrando que la elasticidad del producto de la empresa y el grado de competencia de la industria son variables altamente significativas para explicar dicha interrelación. 
dumbre son importantes para entender el desempeño industrial reciente del país. Según estos autores, Argentina se ha caracterizado por una alta volatilidad y una baja calidad institucional ${ }^{21}$, lo que genera un aumento en la incertidumbre respecto a la evolución futura de la economía y de las inversiones realizadas. En este marco, el horizonte de planeamiento sobre el cual los agentes económicos toman sus decisiones de inversión suele acortarse. Se observa como resultado una conducta renuente a la inversión de largo plazo, lo que, a su vez, resalta la importancia de las políticas públicas tendentes a solucionar la situación. Parecería ser, entonces, que en países con estas características el signo de la relación incertidumbre inversión es predominantemente negativo, sobre todo en el largo plazo, cuando las inversiones pueden verse afectadas por cambios en el contexto, y en los cuales las especulaciones respecto al accionar de los agentes de contralor es fundamental.

Finalmente, es importante diferenciar entre el impacto de la incertidumbre específica de la actividad y la incertidumbre del contexto económico. Este aspecto es particularmente relevante en el caso de la actividad gasífera y petrolera, ya que se trata de actividad con un alto grado de riesgo e incertidumbre implícita, particularmente en el eslabón de exploración y producción. En esta línea algunos trabajos han testeado el impacto de la incertidumbre global y la específica en el caso del petróleo y gas natural en diferentes países, la mayoría de ellos países desarrollados (Favero et al., 1992; Mohn y Misund, 2009). Si bien estos trabaj os presentan algunas diferencias metodológicas y de resultados, suelen ser coincidentes en que el impacto de la incertidumbre global y específica es diferente. Mohn y Misund (2009), por ej emplo, obtienen resultados robustos que relacionan la inversión en forma negativa con la incertidumbre general del mercado financiero, pero que sin embargo reacciona positivamente a la incertidumbre en el mercado petrolero.

\subsubsection{Contexto económico, incertidumbre e inversión hidrocarburífera}

Tal como postula Ramos-Martin (2005), el sistema económico, como todo sistema complejo, presenta un comportamiento caótico con una tendencia intrínseca al desequilibrio. De esta forma, el sistema económico mundial se ha enfrentado y se enfrenta a diferentes crisis, regionales y globales, que lo apartan de una situación de equilibrio y que tienen impactos sobre cada uno de los distintos sectores económicos.

De acuerdo con la IEA (2009), el contexto de crisis al que se enfrentó el sistema económico mundial desde finales del año 2007 impacta a la inversión en infraestructura energética por tres vías diferentes: el aj uste de créditos como forma de financiamiento; la disminución en la rentabilidad de las empresas en relación con la rentabilidad alcanzada en el período anterior, como resultado de la disminución en los precios de los energéticos, las expectativas de los mismos y la caída de la demanda, y la reducción en los requerimientos de inversión de capacidad energética, como resultado de la disminución en el corto plazo de la demanda de energía. Si bien esta repercusión en la inversión energética parece ser

21. Para otros desarrollos sobre el impacto que la debilidad institucional ha tenido en el desempeño del sector energético en Argentina, véase Recalde (2007). similar en todo el mundo y en todos los eslabones de la cadena energética, las características propias de cada país, el contexto institucional, económico y político, así como los rasgos propios de cada eslabón, determinarán en qué medida dicho comportamiento repercutirá sobre la inversión. Naturalmente, la complejidad de la situación es mayor en aquellos sistemas que dependen de recursos energéticos que requieren períodos de inversión prolongados, sea esto por un alto peso en recursos no renovables, sea porque requieren de altas políticas de diversificación del sistema.

Un aspecto de vital importancia es cuál es la repercusión que la crisis económica tiene sobre las inversiones de acuerdo con la relevancia de la región, la dotación de recursos y, particularmente, la reacción de las empresas. La IEA (2009) destaca que, ante similares situaciones, las compañías más pequeñas aj ustan en mayor medida sus niveles de inversión. De acuerdo con las investigaciones de las IEA, Ios planes de inversión de las 50 principales empresas productoras de gas y petróleo presentaron en el año 2009 una reducción del 15,4 \% respecto al año anterior, siendo la reducción mucho menos pronunciada en las principales 25 empresas petroleras que en el resto. El mismo organismo destaca además que las proyecciones de inversión en exploración y producción en el mundo para el año 2009 fueron inferiores en un $21 \%$ a las proyectadas en el año 2008. De igual forma, la dotación del recurso tiene un rol primordial para explicar las reducciones futuras de inversión. De acuerdo con la misma agencia, en aquellas regiones con costos de desarrollo mayores, en cierta parte asociados a la accesibilidad y a la magnitud de las reservas, las inversiones caen de forma más pronunciada.

\subsection{Interacción entre los distintos determinantes}

En función de los aspectos mencionados se presenta en la figura 3 un posible esquema para estudiar el comportamiento de la inversión en el upstream en un país como Argentina. El mismo tiene utilidad para mostrar la interacción entre distintos factores en un país con características similares a las de Argentina, teniendo en cuenta que se trata de una economía pequeña con nula o muy baja repercusión sobre el desempeño de la economía global, y con una baja participación relativa a la dotación de recursos, de forma que su desempeño no afecta los precios internacionales de los energéticos ni las proyecciones globales del sistema.

Este esquema muestra cómo los distintos elementos se combinan impactando de forma positiva o negativa en la inversión. Así, por ejemplo, se observa por los diferentes canales en los que una crisis financiera internacional podría afectar la inversión en el upstream en un país pequeño. El contexto de crisis incrementa la recesión económica mundial, la cual afecta de forma negativa al desempeño de la economía local, aspecto que hace disminuir las proyecciones de demanda energética y aumenta la incertidumbre global del país, haciendo ambos rasgos que se reduzca la inversión en exploración. Las reducciones en la exploración disminuyen la sustentabilidad del sistema energético, lo que repercute al mismo tiempo disminuyendo el desempeño futuro de la economía nacional.

Tal como puede observarse, existen relaciones de retroalimentación entre las variables del sistema, lo que, además, otorga complejidad al proceso de toma de decisiones de inversión. Un aspecto de vital importancia, que merece 


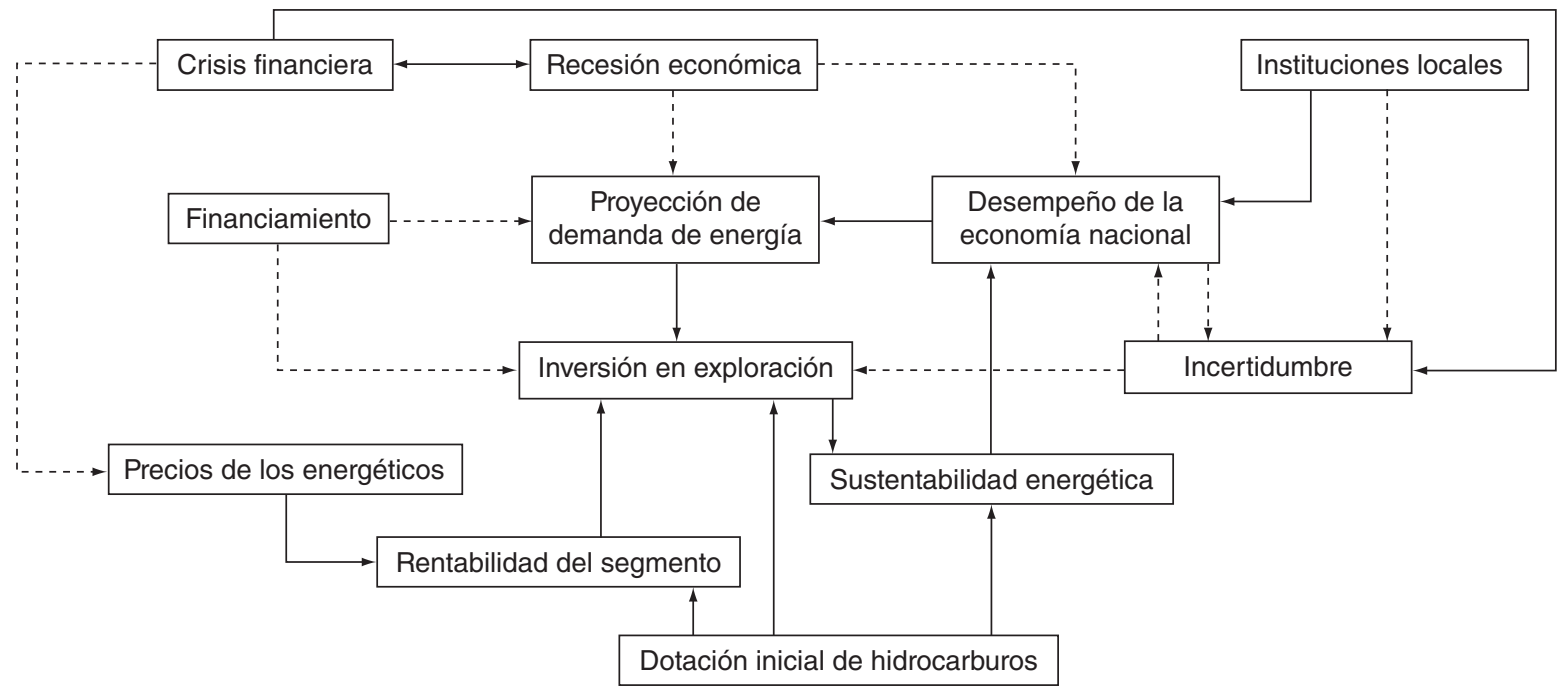

Relaciones positivas _..-- Relaciones negativas

Figura 3 Esquema de interacción entre la inversión en exploración y sus determinantes microeconómicos. Fuente: Elaboración propia.

ser destacado, es el impacto que el contexto de incertidumbre tiene sobre todo el sistema en su conjunto.

\section{Discusión}

El objetivo de este trabajo ha sido analizar la evolución del comportamiento cíclico de la inversión en el upstream de petróleo y gas natural en Argentina, con el fin de bosquejar algún tipo de respuesta en torno a los determinantes de la misma. La discusión principal del análisis ha rondado en torno a la exclusividad o no de los determinantes económicos en la explicación del comportamiento de la inversión. Dada la falta de disponibilidad de series confiables, el análisis ha sido realizado solamente en términos cualitativos, analizando las relaciones entre las variables y desarrollando luego una propuesta de incorporación de variables. Si bien se reconocen problemas en torno a la utilización del número de pozos perforados como proxy de la inversión en exploración en el análisis empírico, esta es en la actualidad la única variable informada por la Secretaría de Energía de la Nación que puede ser utilizada en el análisis.

En primer lugar, los resultados obtenidos en el análisis empírico luego de aplicar a las series el filtro HP no han sido concluyentes para la relación entre las variables en el período bajo análisis en Argentina. Se observa que ninguna de las variables posee un alto grado de poder explicativo, y no se pueden establecer conclusiones fuertes en torno a la fase de desplazamiento y a la dirección del movimiento conjunto de las variables. Nuevamente se reconoce la posible debilidad del análisis no sólo a raíz de la cantidad de los datos utilizados, sino también las posibles diferencias con otros filtros que utilizar, y la falta de información cuantitativa monetaria que permita realizar estudios más pormenorizados. Tal como se mencionara anteriormente, es posible que la riqueza del análisis se incrementara de ser posible realizar estudios con datos de panel a fin de analizar los coeficientes de correlación entre las variables en forma más detallada. Sin embargo, este tipo de estudios se enfrenta con la no disponibilidad de información detallada y de series de tiempo confiables que permita realizar un análisis de panel para el conjunto de empresas operadoras del upstream en el país. De igual forma, la utilización de una variable no monetaria, el número de pozos perforados, como proxy de la inversión, también puede ser responsable de la falta de resultados concluyentes. Nuevamente esto se debe a la falta de disponibilidad de información. Por estos motivos es que se resalta que el análisis ha sido desarrollado solamente a título ilustrativo.

Sobre la base de la falta de solidez explicativa de las variables económicas se avanzó en el análisis teórico de otros determinantes microeconómicos. Entre los principales determinantes se encontrarían: la incertidumbre económica, el desempeño de la economía mundial y local, y la capacidad de control de las instituciones locales, entre otros. Se resalta la relevancia de la interrelación entre dichos factores y el hecho que uno solo de ellos no puede ser analizado como único determinante de la inversión. Del mismo modo, desde la perspectiva de este estudio, el rol de la incertidumbre es fundamental, principalmente por la forma en la que opera sobre los incentivos de las empresas, en particular si las mismas presentan alta adversidad al riesgo.

Así, en el caso de Argentina, como posiblemente de distintos países en desarrollo en los cuales las instituciones se constituyen en los cuellos de botella, se destaca el impacto negativo de la debilidad institucional sobre el diseño de los contratos petroleros y gasíferos al momento de la desregulación petrolera y la privatización del sector, aspecto que posibilitó inicialmente una práctica reticente a la inversión. Más aún con posterioridad a la crisis económica enfrentada a nivel nacional en los años 2001 y 2002, la cual culminó, 
entre otros aspectos, con el congelamiento de las tarifas de los servicios públicos de electricidad y gas natural, el congelamiento del precio de gas a boca de pozo y el establecimiento de un sistema de retenciones a la exportación de petróleo y gas natural (aspecto que implica un diferencial de precios internacionales y locales), se ha desincentivado todavía más la inversión en exploración.

En un sistema energético, el rol del Estado y de las instituciones es fundamental para asegurar el desenvolvimiento sustentable en el tiempo. En el caso de Argentina, la falta de estructuras que otorguen estabilidad al sistema, al igual que la debilidad de los mecanismos de control para asegurar el cumplimiento de los contratos, ha operado en contra de los incentivos a la inversión, quizás en mayor cuantía de lo que lo han hecho los precios de los energéticos.

\section{Agradecimientos}

La autora quiere agradecer especialmente la valiosa ayuda, los comentarios y las sugerencias de la Dra. Mariana Zilio y la Dra. Mara Rojas en la elaboración de este artículo.

\section{Anexo}

Para la estimación de la renta petrolera se ha adoptado el concepto teórico de Renta Absoluta de Monopolio de Marx (1894), siguiendo, entre otros autores, a Mansilla (2006, 2007). Así, la renta petrolera total se define como:

$R T=R M+R D$

Donde:

RT: es la renta petrolera total.

RM: es la renta proveniente del precio de monopolio; muchas veces es denominada por otros autores como renta absoluta o de escasez.

RD: es la renta diferencial proveniente de yacimientos (o pozos) de diferentes calidades o ubicaciones.

Sobre la base de lo desarrollado teóricamente por Marx (1894), se define el precio de producción de los productos hidrocarburíferos y su precio de mercado como:

$$
\begin{aligned}
& p p^{j}=\sum_{i} c_{i}^{j}+\delta \\
& P=p p^{m g}=\sum_{i} c_{i}^{m g}+\delta \\
& \sum_{i} C_{i}^{j}<\sum_{i} C_{i}^{m g} \\
& \mathrm{RD}^{\mathrm{j}}=p \mathrm{p}^{\mathrm{mg}}-\mathrm{pp^{j }} \\
& R D=\Sigma_{j}\left(p p^{m g}-p p^{j}\right) \times \frac{q^{j}}{Q} \\
& p p^{M E}=C m e=\Sigma_{j} p p^{j} \times \frac{q^{j}}{Q} \\
& \mathrm{RD}=\mathrm{pp}^{\mathrm{mg}}-\mathrm{pp}^{\mathrm{ME}} . \\
& \mathrm{RD}=\mathrm{P}-\mathrm{Cme}
\end{aligned}
$$

Donde:

pp: es el precio de producción en el yacimiento j.

$\Sigma c_{i}$ : es la suma de los distintos costos i en el proceso de producción en el yacimiento j.

Se definen como:

$\Sigma_{i} C_{i}^{j}=C_{e x p}^{j}+C_{e x t}^{j}+C_{d e s}^{j}$

Donde:

$\mathrm{C}_{\text {exp }}^{\mathrm{j}}$ : son los costos de exploración en el yacimiento j.

$\mathrm{C}_{\text {ext: }}^{\mathrm{j}}$ : son los costos de extracción en el yacimiento $\mathrm{j}$.

$\mathrm{C}_{\text {des: }}^{\mathrm{j}}$ : son los costos de desarrollo en el yacimiento $\mathrm{j}$.

$\delta$ : es el margen de ganancia normal.

$P$ : es el precio de mercado del producto.

$\mathrm{pp}^{\mathrm{mg}}$ : es el precio de producción en el yacimiento marginal. $\Sigma c_{i}^{\text {ymg: }}$ es la suma de los distintos costos i en el proceso de producción en el yacimiento marginal.

$\mathrm{pp}^{\mathrm{j}}$ : es el precio de producción en el yacimiento j.

$q^{j}$ : es la producción en el yacimiento j.

$\frac{q^{j}}{Q}$ : es la participación relativa de cada yacimiento.

$\mathrm{pp}^{\mathrm{ME}}$ : es el precio de producción medio igual al costo medio.

$\mathrm{RD}^{\mathrm{j}}$ : es la renta diferencial del yacimiento j respecto al yacimiento marginal.

RD: es la renta diferencial total.

Además de esta renta diferencial, existe una renta de monopolio absoluto, la cual se debe a la existencia de precios de monopolio, de forma que:

$\exists P^{M}>P=p p^{m g}$

$\mathrm{RM}=\mathrm{P}^{\mathrm{M}}-\mathrm{pp}^{\mathrm{mg}}$

Los distintos costos en el proceso de producción del yacimiento se definen como la suma de Finding más Lifting Costs. En primer lugar, para la estimación del costo de producción del yacimiento marginal, se han utilizado datos de EIA, que informa los costos de diferentes regiones del mundo. En el caso de Argentina, para el período 1992-1997, se usaron datos de Gadano (1998), quien cita como fuente primaria Ios informes anuales de YPF; para el período 1999-2007, se utilizaron datos de los informes anuales de Repsol YPF. De esta manera, al incluir no sólo los costos de producción del recurso, sino también los de exploración (captados dentro de los finding costs), se realiza una estimación de la renta petrolera y no de la cuasi renta, dato que se obtendría si estos últimos se excluyeran del análisis. El precio de producción (precio de mercado) se estima estableciendo un mark up sobre los costos de producción. Siguiendo a Mansilla (2006, 2007), se supone una rentabilidad del $20 \% 22$.
22. Esta tasa de ganancia puede ser considerada elevada dado que, según Scheimberg (2007), la tasa de rentabilidad de las empresas gasíferas en el país durante 2006 fue del 13,1\% 


\section{Bibliografía}

Abel, A.B., 1983. Optimal investment under uncertainty, American Economic Review 73, 228-233.

André, F., Pérez, J., Martin, R., 2002. Computing white stylized facts on comovement. Economics Letters 76, 65-71.

Asafu-Adjaye, J., 2000. The relation between energy consumption, energy prices and economic growth: time series evidence from Asian developing countries. Energy Economics 22, 615-625.

Bomtempi, M.E., Golinelli, R., Parigi, R., 2010 Why demand uncertainty curbs investment: Evidence from a panel of Italian manufacturing. J ournal of Macroeconomics 32, 218-238.

Bond, S., Van Reenen, J., 2007. Microeconometric models of investment and employment. Handbook of Econometrics $6 \mathrm{~A}$. Elsevier North Holland.

Bouille, D., 2004. Manual de Economía de la Energía. IDEE/ FB, San Carlos de Bariloche.

Boyle, G., Guthrie, G., 2003. Investment, Uncertainty and Liquidity. The journal of Finance 58, 2143-2166.

British Petroleum, 2009. Statistical Review of World Energy. Disponible en: www. bp.com/ statistical/ review

Butzen, P., Fuss, C., Vermeulen, P., 2002. The impact of uncertainty on investment plans. National Bank of Belgium working paper 24.

Caballero, R.J., 1991. On the sign of the investment-uncertainty relationship. American Economic Review 81, 271-288.

Caldwell, B.J ., 1989. Post-Keynesian methodology: an assessment. Review of Political Economy 1, 43-64.

Caruso, N., 2003. Componente: gas natural y derivados. En: Estudios Sectoriales.

Cleveland, C., 2003. Biophysical constraints to economic growth. Encyclopedia of Life Support Systems. Oxford, Reino Unido: EOLSS Publishers Co.

Cleveland, C., Costanza, R., Hall, C., Kaufmann, R., 1984. Energy and the U.S. Economy: A Biophysical Perspective. Science 31, 890-897.

Cleveland, C., Morris, C., 2006. Dictionary of Energy. Elsevier.

Coddington, A., 1982. Deficient Foresight: A Troublesome Theme in Keynesian Economics. American Economic Review 72, 480-487.

De Dicco, R., 2004. Análisis de la participación de los principales conglomerados empresarios en diversas fases de la cadena gasífera y en otros segmentos del mercado energético en Argentina. Material del Área de Recursos Energéticos y Planificación para el Desarrollo 9. IDICSO.-USAL. Buenos Aires.

De Lucas Santos, S. , Álvarez Ayuso, I., Delgado Rodríguez, M., 2009. Análisis de las relaciones entre los ciclos económicos internacionales. Cuadernos de Economía 32, 91-112.

Dixit, A., Pindyck, R., 1994. Investment under uncertainty. Nueva J ersey:

Driver, C., Moreton. D., 1991. The Influence of Uncertainty on UK Manufacturing Investment. Economic J ournal 101, 1452-1459.

Eichnner, A., 1976. The Megacopr and Oligolopy Micro Fudations of Macro Dynamics. Cambridge University Press.

Favero, C., Pesaran, M., Sharma, S., 1992. Uncertainty and irreversible investment, an empirical analysis of development of oilfields on the UKCS. Working Paper (EE17). Oxford Institute for Energy Studies.

Feal Zubimendi, S., Rojas, M., Zilio, M., 2010. Hechos estilizados para la economía argentina. Banco Central de la República Argentina 56, 157-210.

Fischer, M., 2004. Investment in Fossil Fuels Industries. Encyclopedia of Energy 3.

Francis, B., Moseley, L., Osaretin Iyare, S., 2007. Energy consumption and projected growth in selected Caribbean countries. Energy Economics 29, 1224-1232.

Fuss, C., Vermeulen, P., 2004. Firms' investment decisions in response to demand and price uncertainty, ECB Working Paper No. 347.
Gadano, N., 1998. Determinantes de la inversión en el sector petróleo y gas natural de Argentina. Serie reformas económicas 7. Santiago de Chile: CEPAL.

Guiso, L., Parigi, G., 1999. Investment and demand uncertainty. Quarterly J ournal of Economics 114, 185-227.

Guzowski, C., Recalde, M., 2008. Diagnóstico y prospectiva de abastecimiento energético para Argentina. Anales de Ia XLIII reunión anual de la Asociación Argentina de Economía Política.

Hartman, R., 1972. The effects of price and cost uncertainty on investment, J ournal of Economic Theory 5, 258-266.

Hasson, G., Pistonesi, H., 1988. Análisis y modelización del abastecimiento energético. San Carlos de Bariloche: IDEE/ FB.

Hodrick, R.J ., Prescott, E.C., 1980. Post-War US business cycles: an empirical investigation. Discussion Paper 251, Carnegie Mellon University.

IDEE/ FB, 2006. Manual de economía de la exploración. Posgrado en economía y política energética y ambiental. Universidad Nacional del Comahue-Fundación Bariloche.

IEA, 2007. Key world energy statistics.

IEA, 2009. The impact of the financial and economic crisis on global energy investment.

Keynes, J.M., 1936. The General Theory of Employment, Interest and Money. Londres: Macmillan for the Royal Economic Society.

Kosacoff, B., Ramos, A., 2006. Comportamientos microeconómicos en entornos de alta incertidumbre: la industria argentina. Santiago de Chile: CEPAL.

Kozulj, R., 2002. Balance de la privatización de la industria petrolera en Argentina y su impacto sobre las inversiones y la competencia en los mercados minoristas de combustibles. Serie Recursos Naturales e Infraestructura 46. Santiago de Chile: CEPAL.

Kydland, F., Prescott, E., 1982. Time to build and aggregate fluctuations. Econometrica 50, 1345-1371.

Kydland, F., Prescott, C., 1990. Business cycles: real facts and a monetary myth. Federal Reserve Bank of Minneapolis, Quarterly Review, 3-18, Spring.

Lavoie, M., 1992. Foundations of Post-Keynesian Economic Analysis. Edgard Elgar.

Lee, C., 2005. Energy consumption and GDP in developing countries: A cointegrated panel analysis. Energy Economics 27, 415-427.

Mansilla, D., 2006. La renta petrolera en la Argentina (1996-2005). Realidad Económica 223.

Mansilla, D., 2007. Hidrocarburos y política energética. De la importancia estratégica al valor económico: desregulación y privatización de los hidrocarburos en Argentina. Ediciones del CCC Centro Cultural de la Cooperación Floreal Gorini.

Marx, K., [1894] 2001. El capital: crítica a la economía política. Tomo III. México DF: Fondo de Cultura Económica.

Mohn, K., Misund, B., 2009. Investment and uncertainty in the international oil and gas industry. Energy Economics 31, 240-248.

Narayan, P., Smyth, R., 2009. Multivariate granger causality between electricity consumption, exports and GDP: Evidence from a panel of Middle Eastern countries. Energy Policy 37, 229236.

Ogawa, K., Suzuki, K., 2000. Uncertainty and Investment: Some Evidence from the Panel Data of J apanese Manufacturing Firms. J apanese Economic Review 51,170-192.

Oh, W., Lee, K., 2004. Causal relation between energy consumption and GDP revisited: the case of Korea 1970-1999. Energy Economics 26, 51-59.

Ramos-Martin, J., 2005. Complex systems and exosomatic energy metabolism of human societies. Universitat Autònoma de Barcelona.

Recalde, M., 2007. Calidad institucional argentina: influencia sobre el sector energético. Argentina: Anales del tercer Congreso de Estudiantes de Postgrado en Economía (III CNEPE). 
Recalde, M., 2010. Sistemas energéticos y desarrollo socio económico: implicancias del control sobre los recursos naturales energéticos. Universidad Nacional del Sur.

Sari, R., Soytas, U., 2007. The growth of income and energy consumption in six developing countries. Energy Policy 35, 889-898.

Scheimberg, S., 2007. Experiencia reciente y desafíos para la generación de renta petrolera "aguas arriba" en Argentina. Documento de Proyecto, CEPAL.

Secretaría de Energía de la Nación, 2008. Balances energéticos 1990-2007. Buenos Aires.

Serven, L., 1998. Macroeconomic Uncertainty and Private Investment in Developing Countries: An empirical Investigation. Policy Research Working Paper 2035, The World Bank Development Research Group.

Soytas, U., Sari, R., 2003. Energy consumption and GDP: causality relationship in G-7 countries and emerging markets. Energy Economics 25, 33-37.
Sterken, R., Lensink, R., Hong B., 2002. Investment, cash flow and uncertainty: Evidence for the Netherlands'. University of Groningen.

Stern, D., 2009. Interfuel Substitution: A Meta-Analysis. Munich Personal RePEc Archive.

Stern, D., Cleveland, C., 2004. Energy and economic growth. En: Rensselaer Working Papers in Economics 0410.

Van Zon, Y., 2003. An endogenous growth model with energy saving technical change. Resource and Energy Economics 25, 81-103.

WORLD ENERGY COUNCIL (2007). 2007 Global Energy Survey.

Wray, L., Tymoigne, E., 2008. Macroeconomics Meets Hyman P. Mynsky: The financial Theory of Investment. Working Paper 543. The Levy Economics Institute of Bard College.

Zachariadis, T., 2007. Exploring the relationship between energy use and economic growth with bivariate models: New evidence from G-7 countries. Energy Economics 29, 1223-1253. 